\title{
SISTEMAS DE ENFRENTAMIENTO EN FAMILIAS DE ENFERMOS ALCOHOLICOS
}

\author{
SISTEMS OF COPING IN THE FAMILIES OF ALCOHOLICS
}

NAYADE RIQUELME PEREIRA ${ }^{1}$, JOSE MANUEL MERINO ESCOBAR ${ }^{2}$

\begin{abstract}
RESUMEN
Dado el grave problema del alcoholismo en Chile y las alteraciones que provoca en la familia, este estudio se propone identificar y relacionar los estilos de enfrentamiento más uso-efectivos para las familias de los enfermos alcohólicos con variables sociodemográficas y psicosociales.

La muestra estuvo constituida por un familiar de apoyo de cada familia, que totalizaron 46 personas.

Fueron utilizados 6 instrumentos: Cuestionario antecedentes sociodemográficos, Escala de Enfrentamiento (Jalowiec), Cuestionario MOS de Apoyo Social, Escala de Preocupación Empática (Davis), Test de Proactividad (C. Intelig. Emocional, Caracas), Cuestionario de Funcionamiento Familiar (Hernández).

Los estilos de enfrentamiento con mayor nivel de uso-efectividad para estas familias, en orden decreciente, fueron: el estilo optimista, apoyante, confrontativo y autoconfiante.

Estos estilos, en general, fueron desarrollados por personas mayores de 41 años, con escolaridad básica y de situación socioeconómica baja. Podría deducirse, entonces, que estas personas a lo largo de su vida han desarrollado un estilo de vida positivo para convivir y superar la adversidad que los ha rodeado, y que les permite ser apoyo de un familiar que presenta la enfermedad alcohólica.

Se recomienda continuar investigando acerca del sistema relacional de las familias de enfermos alcohólicos en Chile.
\end{abstract}

Palabras claves: Estilos de enfrentamiento, Familias de enfermos alcohólicos.

\begin{abstract}
The alcoholism is one of the most traditional and most prevalent problems in Chile, affecting especially to the family.

The purpose of this study is to identify and relate the coping styles plus effective-use for the families of alcoholics with sociodemographic and psychosocial variables, using Sociodemographic Data Questionnaire, Jalowiec's Coping Scale, MOS Questionnaire of Social Support, Emphaty Scale (Davis), Proactivity Test (C. Emotional Intelligence of Caracas), and Hernandez's Family Functioning Scale.

The sample is made up by one supporting relative from each family totaling 46 people.

The coping styles that resulted in a superior level of effective use for the families, in a decreasing order, were: optimistic style, supportive, confrontational and self-reliant.

These styles, in general, were developed by persons over 41 years old, with primary schooling, and belonging to a low social and economic level, situation that allows us to deduct that all through their lives they have developed a positive style of life in order to co-exist and overcome surrounding adversity thus enabling them to support a relative who is an alcoholic.

It is recommended to continue researching into the system of relations among families of alcoholics in Chile.
\end{abstract}

Keywords: Coping styles, Families of alcoholics.

${ }^{1}$ E.U. Instructor. Dpto. Enfermería. Fac. Medicina. Universidad de Concepción. Candidata a Magíster en Salud Familiar. Universidad de Concepción.

${ }^{2}$ Philosofical Doctor in Sociology. The University of Texas at Austin, USA. Profesor Titular. Facultad de Ciencias Sociales. Universidad de Concepción. 


\section{INTRODUCCION}

Dentro del sector salud uno de los problemas más tradicionales y con mayor incidencia en Chile es el alcoholismo, no sólo por el alto porcentaje de personas afectadas, sino también por las concomitancias involucradas, entre las cuales está la familia.

Los datos estadísticos entregados en el informe ejecutivo del IV Estudio Nacional de Consumo de Drogas en Chile, 2000, del Consejo Nacional para el Control de Estupefacientes (CONACE), establecen una prevalencia global de consumo de alcohol en el último mes del orden del $53.34 \%$, observándose en el sexo masculino un valor considerablemente mayor de prevalencia observada que en el sexo femenino $(62.06 \%$ versus $46.19 \%$, respectivamente), conjuntamente se indica que los tramos intermedios de edad, entre los $19 \mathrm{y}$ 44 años -que corresponde a los llamados adultos-jóvenes-, concentran los mayores porcentajes de población afectada.

Llama la atención, de acuerdo a un informe CONACE anterior, que un $2.92 \%$ de los hombres y un $0.66 \%$ de las mujeres hayan declarado consumo de alcohol entre 26 y 30 días del mes, lo que permite vislumbrar el consumo excesivo, que en los hombres es cuatro veces mayor que en las mujeres y que, seguramente, incluye a una buena proporción de los denominados enfermos alcohólicos. Actualmente el $23.78 \%$ de los encuestados presenta el test EBBA positivo (Escala Breve del Beber Anormal), que hace referencia a las personas que ya están presentando un consumo problemático.

La denominación "enfermedad alcohólica" fue dándose conforme progresaban los criterios diagnósticos para ir reemplazando la catalogación inicial de alcoholismo como un vicio o una figura delictiva, y ser incluida en el capítulo de las enfermedades, alineada en el círculo de las drogadependencias, entendiéndose el alcoholismo como la clase de drogadependencia generada por la droga institucionalizada en el sistema ideológico imperante en la cultura occidental (Madden, 1979).
La noción de enfermedad, indudablemente, sólo puede utilizarse a partir del momento en que se ha establecido la dependencia. La alcoholdependencia puede definirse como el impulso irresistible de consumir alcohol. El individuo no es capaz de contener la necesidad de alcohol. Ha perdido la capacidad de libre determinación para no recurrir a él. Así, tenemos que la alcoholdependencia es el resultado de la interacción entre el sujeto $y$ el alcohol en un cierto contexto sociocultural.

A medida que la disponibilidad de alcohol sea más amplia y su precio más accesible, la tasa de incidencia de la alcoholdependencia y la proporción de sus consecuencias familiares, sociales y laborales se elevan progresivamente.

\section{ALCOHOL Y FAMILIA}

La COFACE, 1999 (Confederación de la Familia para la Comunidad Europea), plantea terminantemente que los problemas con el alcohol en la familia son importantes, porque las familias son importantes.

Las funciones esenciales de la familia son satisfacer las necesidades de sus miembros respecto a la seguridad y bienestar físico, psicológico, social y económico, y proporcionar un ambiente satisfactorio para la mantención, educación y socialización de los hijos (Informe Comisión Nacional para la Familia, 1995). Pero todas estas funciones pueden verse en peligro por el consumo problemático del alcohol.

El alcohólico puede cambiar los papeles desempeñados por los miembros de la familia entre sí y en relación con el mundo exterior. El bebedor problemático puede dejar de desempeñar sus funciones en el hogar, con los hijos, en el ambiente laboral y social, y obliga a que sean asumidos por otro miembro de su familia, a menudo su cónyuge o hijo mayor (Steinglass et al., 1988).

No se debe olvidar que los propios procesos interaccionales de la familia, sobre todo la familia encabezada por un progenitor al- 
cohólico, son los factores responsables de la propagación intrafamiliar de la alcoholdependencia. Se confirma aquí que una enfermedad familiar no es sinónima de una enfermedad hereditaria, sino que se debe, más que nada, a la influencia de la convivencia que a la herencia (Bourgeos y cols., 1975).

Actualmente se va concretando y definiendo cada vez más que la etiología básica del alcoholismo es psicosocial, lo que se sistematiza en una serie de factores concretos de orden psicológico. La influencia alcohólica ejercida por los familiares alcohólicos sobre el niño, por ejemplo, es doble: por una parte, la vida familiar en contacto con un miembro alcohólico, especialmente cuando éste es el padre o la madre, impide muchas veces a los descendientes el desarrollo de hábitos comunicativos y la tolerancia a las frustraciones, conduciéndoles, en definitiva, a la formación personal de los elementos propios y condiciones para desarrollar la enfermedad. Por otro lado, los niños suelen vincularse a los adultos con los que conviven por lazos de identificación, tendiendo a adoptar las mismas pautas de conducta que ellos y, por lo tanto, la conducta alcohólica (Alonso-Fernández, 1992).

Los cambios en el estado de la alcoholdependencia del marido generan en la conducta de la esposa cambios razonables y previsibles. Estos cambios pueden ser utilizados por el marido para racionalizar su entrega a la bebida (James y Goldman, 1971).

Actualmente se hace alusión al fenómeno de la codependencia, atribuido casi exclusivamente a las mujeres, el que se refiere a las maneras con las cuales las parejas compensan o cubren el comportamiento destructivo de sus compañeros bebedores, posiblemente facilitando que ellos sigan bebiendo (Treadway, 1990).

En esta trama familiar, profundamente ambivalente y conflictiva, los hijos forman un grupo compacto con la madre, la coalición madre-hijos, provocando el aumento de los celos del padre, sus frustraciones, sentimientos de exclusión, y aumenta por supuesto la violencia intrafamiliar en todas sus dimensiones (Quiroz M., 1994).

Dentro de las complicaciones y consecuencias familiares del alcoholismo, se encuentra la desintegración de los hogares fundados por alcoholdependientes, la que se sistematiza en una progresiva escala desde el desajuste familiar hasta la disgregación familiar, cruzado permanentemente por un sentimiento de incertidumbre hacia condiciones futuras, tanto en la pareja como en los hijos.

Cuando se analizan todas estas circunstancias que rodean a la familia de enfermos alcohólicos y si nos situamos específicamente en la etapa más difícil de esta enfermedad, en la cual los trastornos conductuales se expresan permanentemente, cabe preguntarse: ¿Qué hace que las familias de enfermos alcohólicos puedan superar el tiempo crítico de esta enfermedad y llegar a vivenciar la recuperación y rehabilitación en conjunto?

Para conocer y comprender esta realidad, se tratará de buscar en la teoría del enfrentamiento datos o variables que nos expliquen las condicionantes, capacidades o destrezas que hacen que la familia de los enfermos alcohólicos pueda superar la etapa crítica de la enfermedad y no claudicar y optar por la separación o el desentendimiento.

Lazarus \& Folkman (1986) definen la teoría del enfrentamiento "como aquellos esfuerzos cognitivos y conductuales constantemente cambiantes que se desarrollan para manejar las demandas específicas externas y/o internas que son evaluadas como excedentes o desbordantes de los recursos del individuo".

Hablar de un proceso de enfrentamiento significa hablar de un cambio en los pensamientos y actos a medida que la interacción va desarrollándose; por lo tanto, el enfrentamiento es un proceso cambiante en que el individuo, en determinados momentos, debe contar principalmente con estrategias, ya sean defensivas o con otras que sirvan para resolver el problema, todo a medida que vaya cambiando su relación con el entorno.

Las variaciones pueden ser el resultado de 
los esfuerzos de enfrentamiento dirigidos a cambiar el entorno o su traducción interior, modificando el significado del acontecimiento o aumentado su comprensión. Lazarus \& Folkman mencionan que lo que se llaman etapas de enfrentamiento pueden ser el simple reflejo de las demandas físicas o ambientales cambiantes y de las correspondientes secuencias internas que tienen lugar en el sujeto.

La función del enfrentamiento tiene que ver con el objetivo que persigue cada estrategia, las funciones no se definen en términos de resultados, aunque cabe esperar que determinadas funciones tendrán determinados resultados.

Janis y Mann (1977) formulan las funciones de enfrentamiento dentro de un marco de decisiones. En su modelo, las funciones principales del enfrentamiento tienen que ver con la toma de una decisión, particularmente la búsqueda y evaluación de información.

White (1974) cita tres funciones para el enfrentamiento: 1) Asegurar la información adecuada del entorno; 2) Mantener condiciones internas satisfechas tanto para la acción como para el procesamiento de la información; 3) Mantener autonomía o libertad de movimientos, libertad para utilizar el propio repertorio según un modelo flexible de expresión.

Lazarus \& Folkman (1986) proponen, que junto a las funciones ya descritas, existe una diferencia entre el enfrentamiento dirigido a manipular o alterar el problema, "enfrentamiento dirigido al problema", y el enfrentamiento dirigido a regular la respuesta emocional a que el problema da lugar, "enfrentamiento dirigido a la emoción".

Kahn y cols (1964) señalan dos grupos principales de estrategias dirigidas al problema: los que hacen referencia al entorno y los que se refieren al sujeto. Están en el primer grupo las estrategias dirigidas a modificar las presiones ambientales, los obstáculos, los recursos, los procedimientos, etc., y en el segundo grupo se incluyen las estrategias encargadas de los cambios motivacionales o cognitivos.
La enfermera Ann Jalowiec, de la Universidad de Loyola (Chicago), propuso en 1981 un instrumento encargado de la medición de las conductas de enfrentamiento que las personas utilizaban ante diferentes estresores de vida. La escala consistía en 40 conductas de enfrentamiento, las cuales eran valoradas en su utilización y en su efectividad, dentro de un formato Likert. En 1984, la misma autora modificó el formato de esta escala aumentando a 60 los ítemes a consultar. Finalizado el estudio, clasificó los métodos en confrontativo (la persona confronta la situación y la resuelve constructivamente), evasivo (presencia de conductas de evitación frente al problema), optimista (enfrentar positivamente el problema), fatalístico (enfrentamiento desesperanzado ante el problema), emotivo (persona expresa y libera emociones), paliativo (tratar de reducir el estrés con acciones alternativas), apoyante (persona busca elementos que le den apoyo espiritual, profesional, para enfrentar el problema) y autoconfiante (la persona depende más de sí mismo que de otros para manejar la situación).

\section{ANTECEDENTES EMPIRICOS}

Captain C. (1989), en su artículo "Family recovery from alcoholism", menciona que el grado en que la familia es hábil para modular las demandas, usar los recursos, implementar efectivas estrategias de enfrentamiento y hacer los ajustes necesarios en sus relaciones pueden determinar la extensión de los efectos del tratamiento y su mantenimiento. Para esas familias, que han trabajado exitosamente juntas para superar los efectos negativos del alcohol, la experiencia llega a ser el mejor catalizador para continuar creciendo, madurando y tener un estilo de vida saludable.

Chang J.K. y Krantz M. (1996) desarrollaron un modelo causal, en el cual presentan que los adultos hijos de alcohólicos que están mejor capacitados para percibir la utilidad del apoyo social tienden a tener más bajo nivel emocional. 
Emshoff J.G. y Price AW (1999) estudiaron el alcance y naturaleza de los problemas de crecer con un alcohólico en casa y las estrategias de tratamiento y prevención primaria provistas, los que incluían componentes básicos de información, destrezas de enfrentamiento dirigidas a la emoción y al problema, y apoyo social y emocional, los cuales fueron positivamente evaluados respecto a una variedad de mediciones aplicadas.

Willoughby D.F., Kee C.C., Demi A. y Parker V. (2000) investigaron la relación entre estilos de enfrentamiento y adaptación psicosocial en la mujer con enfermedad crónica; se utilizó la Escala de Enfrentamiento de Jalowiec (revisada) y la Escala de Adaptación Psicosocial a la Enfermedad. Se obtuvo relación entre una mejor adaptación con el uso efectivo de algunos estilos de enfrentamiento como el confrontativo, apoyante, optimista, autoconfiante y paliativo. Existe una importante relación entre las formas como la mujer enfrenta su enfermedad crónica y su nivel de adaptación psicosocial a la enfermedad.

Krogh V., Trevisan M., Jalowiec A. y cols (1992) investigaron la relación entre estrategias de enfrentamiento y factores de riesgo de enfermedad coronaria. En ambos sexos, el aumento de años de educación fue asociado con el aumento del uso de estrategias de enfrentamiento dirigidas a la solución del problema y a la disminución del uso de resignación y búsqueda de simpatía.

Lindqvist R, Sjoden P.O. (1998) investigaron las estrategias de enfrentamiento y calidad de vida en pacientes con diálisis peritoneal continua. Los principales resultados presentaron que el estilo de enfrentamiento optimista, de acuerdo a la escala de enfrentamiento de Jalowiec, fue el más extensamente usado tanto por hombres como por mujeres y este estilo fue también considerado el más efectivo en términos de tratamiento ante aspectos estresantes de éste.

Vaillant y cols. (1998) investigaron la asociación entre bajo nivel de apoyo social y mortalidad prematura en hombres cuyas edades fluctuaban entre los 50 y 70 años. Los resultados dieron una poderosa correlación entre adecuado apoyo social y salud física, mucha de la asociación entre poco apoyo social y mortalidad puede ser mediado por alcoholismo, tabaquismo y psicopatología premórbida.

\section{PROPOSITO DEL ESTUDIO}

Identificar los métodos de enfrentamiento más efectivos y más utilizados por las familias de enfermos alcohólicos recuperados para superar la etapa crítica de la enfermedad, con el objeto de colaborar y / o sugerir propuestas a las entidades de salud o psicosociales, que se encuentran al servicio de la comunidad, potenciando y reforzando aquellos métodos identificados a través de acciones concretas.

\section{OBJETIVO GENERAL}

1. Identificar los métodos de enfrentamiento más utilizados y más efectivos para las familias de los enfermos alcohólicos recuperados y su relación con variables sociodemográficas y psicosociales.

\section{MATERIAL Y METODO}

Diseño. Estudio retrospectivo y relacional.

Universo. Lo constituirán las familias de los enfermos alcohólicos recuperados adherentes a los centros de recuperación y rehabilitación de la comuna de Concepción.

Muestra. La constituirá un miembro (de apoyo) de cada una de las familias de los enfermos recuperados de la alcoholdependencia, adheridos a los centros de recuperación y rehabilitación de la comuna de Concepción. Los miembros a entrevistar serían el o la cónyuge del recuperado alcohólico, o la madre o padre que viva con él en el mismo hogar, $u$ otro familiar significativo. Dada la impactante 
reducción del número de centros rehabilitadores en esta comuna, se trabajó con el universo en estudio que desearon participar.

\section{Recolección de datos}

Se realizó una encuesta de tipo estructurado al miembro de apoyo de la familia, para aplicar los instrumentos que respaldarán el estudio:

-Cuestionario de antecedentes sociodemográficos de la familia.

-Cuestionario de Enfrentamiento de Ann Jalowiec: Se consulta por los estilos utilizados y por aquellos que le brindaron mejor tratamiento de la situación estresante.

-Cuestionario MOS de Apoyo Social: Permite obtener la percepción de apoyo social que el entrevistado cree haber recibido en determinado momento.

-Indice de Reactividad Interpersonal (IRI) de M.H. Davis. Dimensión Emocional -
Subescala Preocupación Empática.

-Cuestionario Familia y Adolescencia. ¿Cómo es su familia? Subescala de Funcionamiento Familiar:

-Escala de Proactividad.

Previamente, todos estos instrumentos fueron aplicados a 10 personas en una prueba piloto destinada a determinar su nivel de validez, obteniéndose sólo correcciones del lenguaje. También se les calculó el coeficiente alfa de Cronbach para determinar su confiabilidad, dando valores entre 0.71 y 0.96 .

\section{RESULTADOS}

El tipo de enfrentamiento mayormente utilizado por las familias de los enfermos alcohólicos corresponde al estilo optimista, demostrando una tendencia a ser altamente usado, aunque con un valor discretamente mayor a los estilos que le continúan (Tabla $\mathrm{N}^{\mathrm{o}} 1$ )

Tabla No 1

Grado de utilización de tipos de enfrentamiento por familias de enfermos alcohólicos.

\begin{tabular}{|lcccc|}
\hline Tipos de enfrentamiento & Media & D.S. & Min. & Máx. \\
\hline Emotivo & 1.25 & 0.49 & 0.40 & 2.40 \\
Optimista (1) & 2.13 & 0.50 & 1.00 & 2.89 \\
Paliativo & 1.14 & 0.54 & 0.43 & 2.14 \\
Confrontativo (3) & 1.81 & 0.54 & 0.00 & 2.90 \\
Evasivo & 1.45 & 0.51 & 0.69 & 2.77 \\
Fatalístico & 1.76 & 0.94 & 0.00 & 3.00 \\
Apoyante (4) & 1.78 & 0.64 & 0.60 & 3.00 \\
Autoconfiante (2) & 1.87 & 0.59 & 0.86 & 2.86 \\
\hline
\end{tabular}

Fuente: Investigación "Sistemas de Enfrentamiento en Familias de Enfermos Alcohólicos. Comuna de Concepción, Chile".

El tipo de enfrentamiento que resultó ser comparativamente más efectivo para las familias de los enfermos alcohólicos también fue el estilo optimista, con valor ligeramen- te superior a los estilos confrontativo, apoyante y autoconfiante, que demuestra una regular efectividad (Tabla $\left.\mathrm{N}^{\circ} 2\right)$. 
Tabla $\mathbf{N}^{\circ} 2$

Grado de efectividad de tipos de enfrentamiento para familias de enfermos alcohólicos.

\begin{tabular}{|lcccc|}
\hline Tipos enfrentamiento & Media & D.S. & Mín. & Máx. \\
\hline Emotivo & 1.03 & 0.44 & 0.00 & 2.00 \\
Optimista (1) & 1.84 & 0.65 & 0.67 & 2.78 \\
Paliativo & 0.99 & 0.50 & 0.43 & 2.14 \\
Confrontativo (2) & 1.52 & 0.63 & 0.00 & 3.00 \\
Evasivo & 0.94 & 0.49 & 0.08 & 2.23 \\
Fatalístico & 0.78 & 0.66 & 0.00 & 2.25 \\
Apoyante (3) & 1.38 & 0.61 & 0.60 & 3.00 \\
Autoconfiante (3) & 1.38 & 0.71 & 0.29 & 2.57 \\
\hline
\end{tabular}

Fuente: Idem. Tabla N ${ }^{\circ} 1$.

Las conductas más utilizadas y más efectivas por estilo de enfrentamiento se presentan en la Tabla $\mathrm{N}^{\mathrm{o}} 3$.

\section{Tabla $\mathbf{N}^{\circ} 3$}

Resumen

Conductas más utilizadas y más efectivas por método de enfrentamiento.

\begin{tabular}{|c|c|c|c|c|}
\hline Método & $\begin{array}{l}\text { Item más } \\
\text { utilizado }\end{array}$ & Conducta & $\begin{array}{c}\text { Item más } \\
\text { efectivo }\end{array}$ & Conducta \\
\hline Emotivo & 1 & Se preocupó del problema & 1 & Se preocupó del problema \\
\hline Optimista & $\begin{array}{l}50 \\
2\end{array}$ & $\begin{array}{c}\text { Trató de pensar positivamente } \\
\text { Esperó que las cosas } \\
\text { pudieran mejorar }\end{array}$ & 47 & $\begin{array}{l}\text { Pensó en las cosas buenas } \\
\text { de su vida }\end{array}$ \\
\hline Paliativo & 44 & Trató de mantenerse ocupada & 44 & Trató de mantenerse ocupada \\
\hline Confrontativo & 16 & Trató de mantener la & $\begin{array}{l}4 \\
16\end{array}$ & $\begin{array}{c}\text { Pensó en diferentes maneras } \\
\text { para manejar la situación } \\
\text { situación bajo control } \\
\text { Trató de mantener la } \\
\text { situación bajo control }\end{array}$ \\
\hline Evasivo & 58 & $\begin{array}{l}\text { Deseó que el problema } \\
\text { hubiera desaparecido }\end{array}$ & 14 & $\begin{array}{c}\text { Soñó despierta con una } \\
\text { vida mejor }\end{array}$ \\
\hline Fatalístico & 12 & $\begin{array}{l}\text { Aceptó la situación porque } \\
\text { no había mucho que hacer }\end{array}$ & 12 & $\begin{array}{l}\text { Aceptó la situación porque } \\
\text { no había mucho que hacer }\end{array}$ \\
\hline Apoyante & 17 & $\begin{array}{c}\text { Rezó o puso su confianza } \\
\text { en Dios }\end{array}$ & 17 & $\begin{array}{c}\text { Rezó o puso su confianza } \\
\text { en Dios }\end{array}$ \\
\hline Autoconfiante & 19 & $\begin{array}{l}\text { Guardó sus sentimientos } \\
\text { para sí mismo }\end{array}$ & 37 & $\begin{array}{l}\text { Se dijo a sí misma que podría } \\
\text { manejar cualquier problema }\end{array}$ \\
\hline
\end{tabular}

Fuente: Idem. Tabla N ${ }^{\circ} 1$. 
Cabe hacer notar que la conducta más utilizada para toda la escala fue la que corresponde al ítem No 1 = "se preocupó del problema", del estilo de enfrentamiento emotivo. La conducta menos utilizada fue del estilo de enfrentamiento paliativo, ítem $\mathrm{N}^{\mathrm{o}} 34$ = "se tomó un trago para sentirse mejor", que a la vez fue considerada la menos efectiva para enfrentar el problema. La conducta más efectiva resultó ser del estilo apoyante, ítem $\mathrm{N}^{\mathrm{o}} 17$ = "rezó o puso su confianza en Dios".

\section{USO-EFECTIVIDAD DE LOS ESTILOS DE ENFRENTAMIENTO}

Este concepto surge por la necesidad de averiguar acerca de los métodos de enfrenta- miento que realmente cumplen con la condición de ser los más utilizados y ser los más efectivos a la vez, ya que no es una condición establecida al determinar nivel de utilización y nivel de efectividad de los estilos de enfrentamiento de manera separada.

El índice uso-efectividad se obtiene de la multiplicación de las medias del grado de utilización y del grado de efectividad logrado por cada estilo, entregando la valoración desde 0 a 9 (media=3, D.S=0.96).

El estilo de enfrentamiento con mayor nivel de uso-efectividad resultó ser el método optimista con un valor intermedio (4.12) de una puntuación que va desde 0.0 a 9.0 puntos (Tabla $\left.\mathrm{N}^{\mathrm{o}} 4\right)$.

Tabla $\mathbf{N}^{\circ} 4$

Grado de uso-efectividad de estilos de enfrentamiento para familias de enfermos alcohólicos.

\begin{tabular}{|lcccc|}
\hline Variable & Media & D.S. & Mín. & Máx. \\
\hline Emotivo & 1.32 & 0.83 & 0.00 & 3.20 \\
Optimista (1) & 4.12 & 2.04 & 0.67 & 8.02 \\
Paliativo & 1.33 & 1.21 & 0.18 & 4.29 \\
Confrontativo (3) & 2.94 & 1.81 & 0.00 & 8.40 \\
Evasivo & 1.41 & 0.97 & 0.05 & 3.95 \\
Fatalístico & 1.69 & 1.76 & 0.00 & 6.19 \\
Apoyante (2) & 3.49 & 2.08 & 0.36 & 9.00 \\
Autoconfiante (4) & 2.85 & 1.92 & 0.37 & 6.24 \\
\hline
\end{tabular}

Fuente: Idem. Tabla No 1 .

Los estilos que le siguen en orden decreciente son el apoyante, confrontativo y autoconfiante. Analizando los dos estilos más uso-efectivos se observa la relación significativa que se obtuvo con las siguientes variables sociodemográficas y psicosociales propuestas:

El estilo de enfrentamiento optimista se relacionó en mayor magnitud con la variable edad en su categoría mayor de 41 años, con un nivel de significación más allá del 1 x 10.000. También obtuvo alta significancia la relación entre este estilo y el nivel de escolaridad básico $(\mathrm{p}<0.002)$ ya sea completo o incompleto, lo que se contrapone con el estudio de Krogh y col. (1992), en el que se interpreta que a menor educación mayor tendencia a adoptar estrategias fatalísticas y apoyantes, situación que no se proyecta en este estudio. También se relacionó fuertemente este estilo con los años de convi- 
vencia entre el familiar entrevistado y el rehabilitado alcohólico, principalmente en aquellas personas que llevaban más de 28 años junto a la persona con la enfermedad alcohólica $(\mathrm{p}<0.004)$.

Sumado a lo anterior, se obtuvo un valor límite de significancia en la relación con el tamaño del grupo familiar $(\mathrm{p}=0.060)$, presentando una tendencia que indica que cuando la persona se inserta en un grupo de 5 miembros principalmente, o más, es mayor la opción de desarrollar este estilo, probablemente porque se aleja la posibilidad de sentirse solo frente al problema en cuestión, y se comparte mayormente las responsabilidades y roles que se ven alterados por la situación de alcoholismo de uno de sus miembros.

De las variables psicosociales, sólo 2 se relacionaron con el estilo optimista. La primera de ellas es la empatía o preocupación empática ( $\mathrm{p}=0.055)$, y la segunda, con mayor nivel de significancia es el funcionamiento familiar ( $p<0.0002)$, que juntas demuestran una presencia lógica en quienes presentan este tipo de actitud ante los problemas de vida, y que de manera afín explican el porqué se establece en familiares con tan alta cantidad de años de convivencia.

Lindqvist y col. (1998) y Wahl y col. (1999) concuerdan con el presente estudio respecto a que el estilo optimista es el método de enfrentamiento más uso-efectivo para hacer frente a las enfermedades crónicas.

El estilo apoyante es significativamente importante para los familiares de enfermos alcohólicos del sexo femenino ( $p<0.03)$, dado quizás por la naturaleza sociable de las mujeres y la facilidad de movilizar recursos o energía necesarios para enfrentar los problemas. Junto a ello y concordantemente, resultó tener relación con este estilo el contar con algún familiar cercano al sector de residencia $(p<0.0009)$, lo que probablemente explica la relación inversamente significativa que se plantea entre la adopción de este método de enfrentamiento y el pertenecer a grupos comunitarios $(\mathrm{p}<0.02)$.

La relación que se plantea entre el desarrollo del estilo apoyante y el nivel de proac- tividad ( $p<0.02)$, es una de las relaciones esperadas y concordantes en sus planteamientos básicos, ya que un familiar que busca elementos de apoyo, de cualquier tipo, para enfrentar el alcoholismo de un familiar, necesariamente está desarrollando conductas proactivas con el fin de generar mejores oportunidades para sí y su familia.

La influencia del nivel de apoyo social percibido en la adopción del estilo apoyante $(p<0.003)$ también es una relación esperada, ya que explica, en gran medida, la efectividad de este estilo, pues nos dice que las personas encuentran, en su interacción con otros en el entorno, solución o satisfacción de necesidades presentes en su vida al lado de un familiar alcohólico. Lo anterior estaría relativamente relacionado con lo planteado por Vaillant y col. (1998) haciendo referencia al significativo rol del apoyo social en la mantención del bienestar físico, cuya disminución podría estar mediada por problemas psicopatológicos o adictivos propios.

El funcionamiento familiar está relacionado con este estilo en un nivel de significancia límite $(\mathrm{p}=0.059)$, lo cual podría ser explicado tal vez por la lesión que podría sufrir el componente de participación en la solución de los problemas familiares que plantea esta escala, al ser menos fuerte que las variables anteriormente mencionadas (familiar cerca, alta proactividad, alto apoyo social).

\section{CONCLUSIONES}

En general, los estilos de enfrentamiento más uso-efectivos para las familias de enfermos alcohólicos son estilos activos, movilizadores de recursos o energía, que los hace salir del "status quo" del problema. Las personas que utilizan estos estilos, aparte de estar ubicados en un nivel socioeconómico desde mediobajo hasta marginal, poseen características personales determinadas, como ser mayores de 41 años, con escolaridad básica, y actitudes constructivas y proactivas, de lo que podría deducirse que estas personas han desarrollado un estilo de vida positivo para tratar de 
vivir y superar la adversidad que los rodea, lo cual se ve potenciado por los efectos de la tradición en cuanto a la fortaleza de la institución familia.

El hecho que no haya habido alguna relación fuerte entre los estilos de enfrentamiento más uso-efectivos en este estudio y la variable edad en la categoría menor de 41 años, se podría interpretar como que la generación de familiares más jóvenes está realmente influenciada por la cultura moderna individualista y desvalorizada, que no le permite aceptar la posibilidad de ser apoyo en la enfermedad alcohólica de un familiar.

Se hace necesario concientizar a trabajadores de la salud o sociales a detectar y hacer presente este problema en las prioridades de las instituciones oficiales, ya sea de salud o sociales, y a no perpetuarlo en nuestra sociedad como una condición de vida pseudonormal, para lo cual se debe disponer de programas o actividades que estén basados en estilos que aportan a la resolución del problema como los detectados en este estudio.

\section{REFERENCIAS BIBLIOGRAFICAS}

Alonso-Fernández, F. Alcoholdependencia. Personalidad del alcohólico, $3^{\circ}$ edición. Ediciones Científicas y Técnicas, S.A. Masson y Salvat. 1992. 256 pp. Barcelona, España.

Antonucci, T.; Jackson, J. Cap. 9: “Apoyo social, eficacia interpersonal y salud: Una perspectiva del transcurso de la vida", en Carstensen, L., Edelstein, B.: Intervención psicológica y social. Gerontología clínica. $2^{\circ}$ edición. Ediciones Martínez Roca S.A., pp. 129-148. Barcelona, España.

Berger, P. The war over the family. Penguin Books. 1983

Bourgeois, M., y cols. "Les enfants d'alcooliques. Une enquete sur 66 enfants d'alcooliques d'un service pedosychiatrique", Ann. Med. Psychol. 133: 592609.1975.

Carmona, A. "Transmisión intergeneracional del alcoholismo", libro Resumen $2^{\circ}$ Congreso Nacional de Alcoholismo. Ministerio de Salud-Universidad de Chile. Santiago. Chile. 1986.

Captain, C. "Family recovery from alcoholism. Mediating family factors". Nursing Clinic North American. Mar; 24 (1), pp. 55-57. 1989. USA.

Chang, J.K.; Krantz, M. "Personal and environmental factors in relation to adjustment of offspring of alcoholics". Substance-Use-Misuse. Aug. 31 (10), pp. 1401-1412. 1996. USA

Clair, D.; Genest, M. "Variables associated with the adjustment of offspring of alcoholic fathers". I. Study of Alcohol. 48 (4), pp. 345-355. 1987.

Comunidad de Madrid. Agencia Antidroga: Problemas por el al alcohol en la la familia DELOS S.L. 1999. España.

Consejo Nacional para el Control de Estupefacientes. Informe Final 1996. Stgo. Chile. 1996.

Consejo Nacional para el Control de Estupefacientes. Informe Ejecutivo 2000. Stgo. Chile, 2001

Collins, E.; Jalowiec, A.; White-Williams, C. "Spouse stressors while awaiting heart transplantation". Heart and Lung. Vol. 25. $\mathrm{N}^{\mathrm{o}}$ 1. January-February. 1996.

Cronqvist, A.; Klang, B.; Bjorvell, H. "The use and efficacy of coping strategies and coping styles in a Swedish sample". Quelity of Life Res. Jan; 6 (1): $87-$ 96. 1997. Sweden.

Davis, M.H. Measuring Individual Differences in Empathy. Evidence for a multidimensional

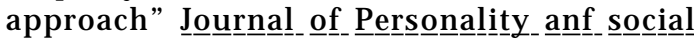
Psychology, 44 (1). 1983.

De la Revilla, L. Conceptos e instrumentos de la atención familiar. Ediciones DOYMA. 180 págs. Barcelona, España

Emshoff, JG.; Price, AW. "Prevention and intervention strategies with children of alcoholics". Pediatrics. May. 103 (5 Pt 2): pp. 1112-1121. 1999. USA.

Gurklis, J.; Menke, E. “Identification of stressor and use of coping methodos in cronic hemodialysis patients" Nursing Research. Vol. 37. N ${ }^{\circ}$ 4. JulyAugust. 1988.

Gulick, E. "Coping among spouses or significant others

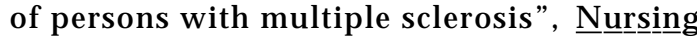
Research. Vol. 44. N 4, July- August. 1995.

Hernández A. Familia y adolescencia. Indicadores de salud. Programa Salud Integral del Adolescente. OPS-OMS. Dic. 1996.

Jalowiec, A.; Powers, M. "Stress and coping in hipertensive and emergency room patients" Nursing Research. Vol. 30. N ${ }^{\circ}$ 1. January-February. 1981.

James, J.; Goldman, M. “Behavior trends of wives of alcoholics" Quart. I. Stud. Alc. N ${ }^{\mathrm{o}} 32$, pp. 373-381. 1971.

Krogh, V.; Jalowiec, A. et al. "Coping and blood pressure". Journal Humane Hypertension. Feb. 6 (1): 65-70. 1992. USA.

Lazarus, R.; Folkman, S. Estrés y procesos cognitivos. $2^{\circ}$ edición. Ediciones Martínez Roca S.A. 463 pp. Barcelona. España. 1986.

Lindqvist, R., Sjoden, PD. “Coping strategies and quality of life among patients on continuous ambulatory peritoneal dialysis". Lournal Adv Nursing. Feb. 27 (2), pp. 312-319. 1998. England.

Madden, J.S. "Commentary on Shaw". Br. Journal Addiction. 74:349-352. 1979. 
Merino, J. "Apoyo social, concepto y medición". $\underline{\text { Rev. }}$ Ciencia y Enfermería. Vol II. No 1. 1996. Chile.

Moos, R.H. "Why do some people recover from alcohol dependence, whereas others continue to drink and become worse over time? Addiccion 89 (1) 3134.

Murphy, S.; Jalowiec, A.; Powers, M. "Psychometric Evaluation of the Hemodialysis stressor scale" Nurs $\underline{\text { ing }}$ Research. Vol. 34. N $\mathrm{N}^{\mathrm{o}}$ 6. NovemberDecember. 1985.

Murray, BL. "Perceptions of adolescents living with parental alcoholism" J. Psychiatric Mental Health Nursing. 5 (6), 525-534. 1998.

Myors, K.; Johnson, M.; Lamgdom, R. “Coping styles of pregnant adolescents" Public Health Nursing. 18 (1), 24-32. 2000.

Peirce, RS; Frone, M.R., Russell, M.; Cooper, ML. “A longitudinal model of social contact, social support, depression, and alcoholic use" Health Psychology. 19 (1), 28-38. 2000.

Quiroz, M. En Ponce, O. Alcoholismo, tabaquismo y otras drogas. Universidad de Concepción. Concepción. Chile. 1994.

Ponce de Luca, R.; Inostroza, R. "Alcoholismo e interacción familiar", libro Resumen $2^{\circ}$ Congreso Nacional de Alcoholismo. Ministerio de Salud-Universidad de Chile. Santiago. Chile. 1986.

Prieto, L. "Estilo y efectividad del enfrentamiento de las esposas de los pacientes con infarto agudo al miocardio" Rev. Ciencia y Enfermería. Vol IV. № 1 . 1998. Chile.

Russell, W.; Booth, B.; Reed, D.; Laughlin, PR. "Personality, social networks, and perceived social support among alcoholics". Journal of Personality. Sep. 65 (3) pp. 649-692. 1997. USA.
Solomon, P.; Draine, J. “Subjetive burden among family members of mentally ill adults: relation to stress, coping, and adaptation". American Journal of Orthopsychiatry. Jul. 65 (3). pp 419-427. 1995. USA.

Steinglass P. et al. The alcoholic family Hutchinson Education, 1988.

Treadway D.: "Codependency:disease, metaphor or fad?". Family Therapy Networker 14 (1). 1990.

Uehara, T.; Sakado, K.; Sakado, M.; Sato, T.; Someya, T. "Relationship between stress coping and personality in patients with major depressive disorder". Psychotherapy Psychosom. 68 (1) pp. 2630. 1999. Switzerland.

Vaillant, G.E.; Meyer, S.E.; Mukamal, K.; Soldz, S. “Are social support in late midlife a cause or a result of successful physical ageing? Psychol-Med. Sep; 28 (5) pp. 1159-1168. 1998. USA.

Vaux, A. Soccial Intervention". Library of Congree Cataloging-inPublication Data. $1^{\circ}$ edición, 1988. U.S.A.

Wahl, A.; Moum, T.; Hanestad, BR.; Wiklund, I.; Kalfoss, $\mathrm{MH}$. "Adapting the Jalowiec Coping Scale in Norwegian adult psoriasis patients". Quality of Life Res. Aug; 8 (5): 535-545. 1999. Norway.

Ward, M. and Faillance, G. "The alcoholic and his helpers". Quart. J. Stud Alc. No 31. 684-691. 1970.

Wikoff R., Miller P. "Analysis of the Jalowiec Scale with cardiac patients'Spouses" Nursing Research. Vol. 38 N4. July- August. 1989.

Willoughby, D.F., Kee, C.C.; Demi, A.; Parker, V. "Coping and psychosocial adjustment of women with diabetes". Diabetes Education. Jan-Feb; 26 (1): pp. 105-112. 2000. USA. 